DOI: $10.24888 / 2500-1957-2020-3-25-35$

УДК $377 ; 378$

\section{СТРАТЕГЕМЫ ПРОЕКТИРОВАНИЯ ПРОГРАММ ЦЕЛЕВОЙ ПОДГОТОВКИ В СОВРЕМЕННОМ ВУЗЕ}

\author{
Татьяна Юрьевна Дорохова \\ к.П.н., доцент \\ tandor81@mail.ru \\ г. Тамбов \\ Николай Петрович Пучков \\ д.П.н., профессор \\ puchkov_matematika@mail.ru \\ г. Тамбов
}

\begin{abstract}
Аннотация. В данной работе рассматриваются вопросы использования методов динамической вариативности при проектировании дополнительных профессиональных программ (ДПП) в вузе, осуществляющем целевую подготовку специалистов. Процесс проектирования представлен как последовательность действий по пошаговому глубокому изучению ситуаций и позиций всех субъектов, заинтересованных в результатах. Цель статьи - на основе анализа проблем современного производства предложить конкретные рекомендации по теории и практике проектирования ДПП в условиях непрерывного изменения производственных заданий.
\end{abstract}

Результаты анкетирования сотрудников предприятий выявили организационные и образовательные проблемы повышения качества целевой подготовки специалистов, и намечены пути их разрешения. Для выработки рекомендаций применялись методы экспертных оценок и вероятностные методы.

Авторами установлено, что обеспечение качества целевой подготовки в вузе достигается за счет разработки ДПП на основе: разбиения процесса проектирования на определенные этапы; использования разноуровневой методологической структуры проектирования; принципа динамической вариативности проектирования; итерационного алгоритма последовательного привлечения экспертов и байесовского подхода для переоценки результатов экспертизы с целью выработки обоснованного оптимального решения.

Разработка образовательных программ должна соответствовать запросам динамично развивающейся экономики, воплощая идеи интеграции науки, образования и производства. Практическая значимость проведенного исследования состоит в обеспечении возможности использования предлагаемой методики разработки ДПП целевого обучения специалистов для наукоемких производств военно-промышленного комплекса, функционирующих в режиме их постоянного обновления. Предложенные рекомендации будут способствовать формированию профессиональных компетенций у профессорско-преподавательского состава и образовательных компетенций у обучающихся и могут быть полезны как для специалистов в области образования, так и для работников промышленных предприятий. Развитием проведенных исследований может стать разработка системы автоматизации процесса проектирования на основе использования новых информационных технологий. 
Ключевые слова: целевая подготовка, дополнительные профессиональные программы, стратегемы, методология поэтапного вариативного проектирования образовательных программ.

Работа выполнена при финансовой поддержке РФФИ (проект № 20-08-00091).

\section{Введение}

В последние годы в стране все отчетливее наблюдаются социально-экономическими изменения, которые заметно сказываются на производственной, научной сферах и на системе образования, их нельзя не учитывать в деятельности даже отдельных организаций и предприятий. Государством приоритетно финансируются целевые программы и проекты массовой цифровизации всех сфер человеческой деятельности. На производстве осуществляется переход на профстандарты, в системе высшего образования наблюдаются процессы фрагментного обновления государственных образовательных стандартов подготовки специалистов, в вузах постоянно перерабатываются действующие учебные планы, рабочие программы, разрабатываются дополнительные образовательные программы, в соответствии с потребностями региональной экономики. По ряду направлений подготовки специалистов организуется целевой прием студентов как с целью повышения престижности отдельных отраслей производства (например, для оборонно-промышленного комплекса $(\mathrm{OПК)),} \mathrm{так} \mathrm{и} \mathrm{для} \mathrm{обеспечения} \mathrm{квалифицированными} \mathrm{кадрами} \mathrm{регионов,} \mathrm{имеющих} \mathrm{кадровый}$ дефицит. Целевая подготовка становится важнейшим показателем воплощения идеи (и программных документов) интеграции науки, образования и производства.

Традиционная система образования, ориентированная на формирование отдельных (базовых) компетенций, не в состоянии удовлетворить потребности современного общества в целевых специалистах. Требуется поиск новых форм и подходов к организации образовательного процесса, обеспечения единства теоретико-методологических основ проектирования различного рода профессиональных образовательных программ, их интеграции. Все это находит свое отражение в новых образовательных стандартах, учебных планах и образовательных программах. Ключевая роль в вопросах целевой подготовки отводится созданию именно региональной системы профессиональной подготовки таких специалистов, так как территориальная обусловленность вузов ориентирована на подготовку специалистов для предприятий своего региона. При этом в процессах проектирования новых образовательных программ оказывается задействованным более широкий круг различного рода специалистов, которых необходимо вооружить методологией педагогического проектирования.

Федеральный закон «Об образовании в Российской Федерации» от 29 декабря 2012 года № 273-Ф3 фактически не регламентирует содержание дополнительных программ профессионального образования, поэтому многие методические аспекты их проектирования в образовательных организациях нормативно не закреплены, хотя положения порождают ряд существенных проблем.

Организация целевой подготовки специалистов в Тамбовском государственном техническом университете (ТГТУ) берет свое начало в 1993 году и ориентирована на комплексное удовлетворение требований всех субъектов, заинтересованных в целевой подготовке обучающихся, их родителей, государства, предприятия-заказчика и вуза. С 2014 года в рамках ведомственной целевой программы «Развитие интегрированной системы обеспечения высококвалифицированными кадрами организаций оборонно-промышленного комплекса РФ (ВЦП Новые кадры ВПК) в вузе ведется подготовка по 18-ти специализированным образовательным программам. При этом вуз является системообразующим звеном целевой подготовки в регионе [1]. 
Первостепенной задачей при этом является качественная разработка ДПП целевой подготовки. В последнее десятилетие для предприятий радиоэлектронного профиля г. Тамбова характерны следующие преобразования: оснащение современной аппаратурой, оборудованием; повсеместная цифровизация производственных процессов; использование новых компьютерных программ, новых форм организационной работы; реструктуризация и перераспределение штатных сотрудников и многое другое. По мнению администрации и сотрудников, это привело, с одной стороны, к повышению производительности труда, а с другой, — к увеличению производственной нагрузки на одно физическое лицо в 1,5-2 раза (в основном из-за необходимости освоения нового).

В данной статье на основе анализа данных экспериментальных исследований условий труда работников предприятий-заказчиков и потребностей молодых специалистов, выявляются причины наличия основных профессиональных трудностей на рабочем месте, потребности в разработке программ целевой подготовки и их содержательном наполнении; описаны возможные методики разработки и реализации целевых программ, предлагается авторский подход к проектированию вариативных образовательных программ целевой профессиональной направленности.

\section{Материалы и методы}

Первая проблема разработки ДПП - проблема их содержательного наполнения. В рамках проектирования ДПП целевой подготовки специалистов радиоэлектронного профиля в 2015-2019 гг. нами проведено экспериментальное исследование, в котором приняли участие работники организаций оборонно-промышленного комплекса г. Тамбова. Первое, что уже привлекло внимание, - это малая доля молодых сотрудников (21\% в возрасте до 35 лет), что недостаточно для передачи опыта и знаний от старшего поколения работников молодежи (воспроизводства кадров). В то же время многие из этого контингента, не имея высшего образования, занимают инженерные должности. В то же время переход на профстандарты затрагивает вопросы установления соответствия работника виду занимаемой должности определенному виду осуществляемой им профессиональной деятельности.

Нами анализировались причины медленного освоения новых производственных заказов, в большинстве случаев - это увеличение объемов специальных индивидуальных заказов, требующих определенного сопровождения как на документальном, так и производственно-техническом уровне, что согласуется с данными зарубежных авторов.

Кроме того, на эффективность работы предприятий радиоэлектронного профиля отрицательно влияют следующие факторы:

- отсутствие должного кадрового обеспечения;

- несовершенство системы оплаты труда.

Кроме того, следует учитывать, что современные работники предприятий должны иметь навыки инновационной деятельности. Опыт подготовки молодых специалистов и (их наставников) к такой деятельности практикуется в нашем вузе сравнительно давно [6]. Первостепенной задачей было выявление портрета современного специалиста. С этой целью осуществлялась оценка эффективности функционирования различных отделов и подразделений, исследовались особенности профессиональной деятельности и видов решаемых задач. В качестве количественных показателей эффективности изучались затраты рабочего времени на различные виды профессиональной деятельности в течении одной рабочей недели. Конечной целью стояла задача выявить дополнительную группу специализированных профессиональных компетенций, на которые должна быть ориентирована целевая подготовка.

Анкетирование сотрудников предприятий и ведущих специалистов выявило наиболее значимые причины, влияющие на эффективность производства (структурированные в Таблице 1). 
Таблий 1.

Причинь, влияющие на эффективность производства

\begin{tabular}{|l|c|}
\hline \multicolumn{1}{|c|}{ Варианты ответов } & $\begin{array}{c}\text { \% ответивших } \\
\text { респондентов }\end{array}$ \\
\hline $\begin{array}{l}1.1 \text { Увеличение объемов профессиональной нагрузки, в результате } \\
\text { возросшего документооборота }\end{array}$ & 79 \\
\hline $\begin{array}{l}1.2 \text { Возросший объем сопроводительной документации при } \\
\text { производстве продукции }\end{array}$ & 46 \\
\hline \begin{tabular}{l}
1.3 Недостаточное количество специалистов в подразделениях \\
\hline $\begin{array}{l}1.4 \text { Нерациональную организацию труда в отделах и структурных } \\
\text { подразделениях }\end{array}$
\end{tabular} \\
\hline
\end{tabular}

С производственной нагрузкой оказалось доступным справиться подавляющему большинству опрошенных - 95\%; в то же время $65 \%$ из них ответили, что повышенная нагрузка, хотя и незначительно, но снижает качество выполняемой ими работы и только $35 \%$ уверенно ответили, что нагрузка не влияет на качество производимых работ.

Следующая часть исследования касалась оценки фактических затрат рабочего времени на виды профессиональной деятельности при решении основных профессиональных задач (Таблица 2). В дальнейшем это явилось исходной информацией, чтобы упорядочить и выделить группу специализированных профессиональных компетенций и их компонентную структуру.

Таблииа 2.

Затраты рабочего времени при решении профессиональных задач

\begin{tabular}{|c|c|}
\hline Индикаторы компетенций & $\begin{array}{l}\text { \% затрат } \\
\text { рабочего } \\
\text { времени }\end{array}$ \\
\hline \multicolumn{2}{|l|}{ 1. Научно-исследовательская деятельность } \\
\hline 1.1 Работа на компьютере, оформление документации & 40 \\
\hline 1.2 Работа с нормативной документацией, справочниками и литературой & 30 \\
\hline 1.3 Консультации и обсуждения с коллегами сложных вопросов & 20 \\
\hline 1.4 Работа по изучению опыта коллег, поиск новых творческих решений & 5 \\
\hline 1.5 Самоконтроль и проверка выполненной работы & 5 \\
\hline \multicolumn{2}{|l|}{ 2. Организационно-управленческая деятельность } \\
\hline 2.1 Работа на компьютере и оформление отчетной документации & 40 \\
\hline $\begin{array}{l}2.2 \text { Обсуждение текущих вопросов } \\
\text { планирование и распределение задач }\end{array}$ & 40 \\
\hline 2.3 Самопроверка выполненной работы & 5 \\
\hline 2.4 Проверка и оценка проделанной работы коллегами & 15 \\
\hline \multicolumn{2}{|l|}{ 3. Проектно-технологическая деятельность } \\
\hline $\begin{array}{l}3.1 \text { Работа на компьютере по проектированию и составлению проектно- } \\
\text { технологической документации }\end{array}$ & 60 \\
\hline $\begin{array}{l}3.2 \text { Работа с нормативно-технической документаций, стандартами и } \\
\text { профессиональной литературой }\end{array}$ & 5 \\
\hline 3.3 Консультации сотрудников других & 15 \\
\hline 3.4 Самоконтроль выполненной работы и сдача отчетной документации & 5 \\
\hline
\end{tabular}




\begin{tabular}{|c|c|}
\hline Индикаторы компетенций & $\begin{array}{l}\text { \% затрат } \\
\text { рабочего } \\
\text { времени }\end{array}$ \\
\hline $\begin{array}{l}3.5 \text { Работа с коллегами по обсуждению текущих вопросов и заданий, } \\
\text { планирование работ }\end{array}$ & 5 \\
\hline \multicolumn{2}{|l|}{ 4. Сервисно-эксплуатационная деятельность } \\
\hline 4.1 Визуальный и инструментальный осмотр объектов & 20 \\
\hline $\begin{array}{l}4.2 \text { Подготовка оборудования и проведение исследований узлов и деталей на } \\
\text { компьютере }\end{array}$ & 20 \\
\hline 4.3 Работа с документацией & 20 \\
\hline 4.4 Подготовка систем и стендов для испытаний и настройки аппаратуры & 5 \\
\hline 4.5 Исследование исправности оборудования & 25 \\
\hline 4.6 Самоконтроль проделанной работы & 5 \\
\hline 4.7 Консультации коллег-сотрудников & 5 \\
\hline
\end{tabular}

Результаты исследования высветили наиболее трудоемкие виды деятельности, это: моделирование, проектирование и настройка комплексов радиосвязи, работа в команде, выполнение исследовательских научных и инновационных проектов.

На основе проведенных исследований был определена компетентностная модель (портрет современного специалиста), представленная в Таблице 3.

Таблица 3

Компетентностная модель - портрет современного специилиста при цุелевом обучении

\begin{tabular}{|c|c|}
\hline \multicolumn{2}{|r|}{ Компоненты компетенций } \\
\hline Профессиональная компонента & ьная компонента \\
\hline $\begin{array}{l}\text { Специализированные } \\
\text { профессиональные } \\
\text { компетенции }\end{array}$ & $\begin{array}{c}\text { (универсальные и } \\
\text { общепрофессиональные } \\
\text { компетенции) }\end{array}$ \\
\hline $\begin{array}{c}\text { Научно- } \\
\text { исследовательские }\end{array}$ & $\begin{array}{l}\text { Структурные компоненты компетенций } \\
\text { - мотивационно-ценностный (профессиональные мотивы и } \\
\text { интерес к профессии, готовность работать по специальности, к } \\
\text { профессиональному развитию); }\end{array}$ \\
\hline $\begin{array}{l}\text { Проектно- } \\
\text { технологические/ Design } \\
\text { and technological }\end{array}$ & 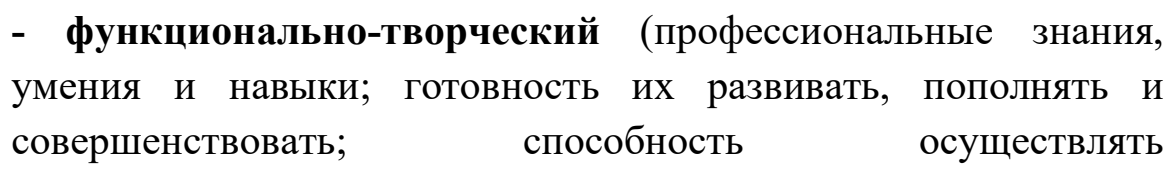 \\
\hline $\begin{array}{c}\text { Сервисно- } \\
\text { эксплуатационные/ } \\
\text { Service and maintenance }\end{array}$ & $\begin{array}{l}\text { профессиональную деятельность; } \\
\text { - рефлексивно-оценочный (способность самостоятельно } \\
\text { принимать решения и брать на себя ответственность; } \\
\text { способность критически оценивать других; объективная } \\
\text { самооценка) } \\
\text { - коммуникативный (коммуникабельность; способность к } \\
\text { работе в команде; знание норм и правил этики поведения в } \\
\text { коллективе }\end{array}$ \\
\hline
\end{tabular}

Выявлены основные умения работников, востребованные современным производством: коммуникативного взаимодействия; осуществлять контроль и самоконтроль выполняемых работ; способности принимать нестандартные решения и креативные подходы к решению профессиональных задач. 
Реализованная методика исследования позволила выявить, структурировать, обосновать наличие компетенций, определяющих содержание профессиональной деятельности специалистов для предприятий радиоэлектронного профиля, на которую должны быть ориентированы ДПП целевой подготовки.

\section{Результаты исследования}

Специфические сложности построения (разработки) ДПП определяются наличием конкретных факторов, многие из которых явно не измеримы, поэтому не поддаются математической обработке и обеспечению возможности выбора, таким образом, оптимальной ДПП. Существенно влияет и региональная привязанность: разработка такого рода программ осуществляется в условиях прямого непосредственного участия практически всех субъектов, заинтересованных в результатах их реализации, (обучающие, обучаемые и их родители, заказчики, отрасль, государство), поэтому решаются не просто проблемы экономики, дидактики высшей школы, но и многочисленные организационнообразовательные проблемы: формы обучения, конкретного места занятий, графика учебных занятий, административного руководства учебным процессом и т.п., т.е. должны выполняться условия вариативных образовательных программ [5], программ, отвечающих целям учета национальных, региональных и местных социо-культурных особенностей и традиций, обеспечивающих индивидуальный характер развития обучающихся в соответствии с их склонностями и интересами.

Учитывая то обстоятельство, что проведение педагогических профессиональных экспериментов с целью проверки обоснованности выдвигаемых идей и предложений требует больших временных затрат, то принятие оперативных решений по содержанию, структуре, условиям реализации ДПП, целенаправленных на своевременную реализацию инновационных проектов, наиболее реально осуществлять на основе экспертных оценок различных вариантов специалистами, используя методы научного планирования и методы статистической обработки полученных результатов. Опыт нашей работы позволяет утверждать, что реально объективным можно считать идеологию стратегем.

Термин «стратегема» в нашем исследовании означает, прежде всего, выбор методологических основ проектирования ДПП, обеспечивающих достижение требуемого качества целевой подготовки специалистов с учетом социально-экономической ситуации и психологических особенностей как обучающихся, так и заказчиков специалистов.

Учитывая динамично меняющийся характер профессиональных задач, отражающихся в конкретных целевых результатах [3], наиболее целесообразным, на наш взгляд, стало применение в качестве первой стратегемы - идеи поэтапного проектирования [4], в соответствии с которой целостный процесс проектирование ДПП целевой подготовки разбивается на совокупность нескольких этапов, на каждом из которых вся совокупность действий подвергается оценочному анализу, с учетом возникающих новых условий и имеющихся вариантов решений, а каждый шаг строится на оценке возможных следствий и возможных субъективных противодействий. При этом основными задачами проектирования, с методической точки зрения, считаем следующую последовательность действий: мотивацию (раскрытие актуальности проекта); формирование концепции (решения существующих проблем социально-экономического развития); непосредственное создание проекта, его реализация (внедрение); проверку адекватности решению поставленных задач, оценку эффективности [2]. Результаты решения каждой из перечисленных задач необходимо предопределяют выполнение последующих, что обеспечивает, таким образом, выполнение принципа непрерывности процесса проектирования.

В результате анализа результатов предпроектных исследований в качестве второй стратегемы нами выбрана - трехуровневая методология проектирования, реализуемая в четыре этапа и опирающаяся на теоретико-методологические подходы и методы (Таблица 4). 
Таблица 4.

Стратегема методологии поэтапного проектирования

\begin{tabular}{|c|c|c|c|c|}
\hline \multicolumn{5}{|c|}{ Методологические уровни поэтапного проектирования ДПП } \\
\hline Уровни & Целевой & Функционально- & Оценоч & ный \\
\hline $\begin{array}{l}\text { Теоретико- } \\
\text { методологи } \\
\text { ческие } \\
\text { подходы }\end{array}$ & $\begin{array}{l}\text { Компетентност } \\
\text { ный, } \\
\text { системный, } \\
\text { процессный, } \\
\text { праксиологиче } \\
\text { ский }\end{array}$ & $\begin{array}{l}\text { Интегративно- } \\
\text { модульный, } \\
\text { системный, } \\
\text { средовый, } \\
\text { личностно- } \\
\text { ориентированный }\end{array}$ & $\begin{array}{l}\text { Квалиметрически } \\
\text { й, } \\
\text { синергетический, } \\
\text { процессный }\end{array}$ & $\begin{array}{l}\text { Синергетичес } \\
\text { кий, } \\
\text { квалиметриче } \\
\text { ский, } \\
\text { кибернетичес } \\
\text { кий }\end{array}$ \\
\hline $\begin{array}{l}\text { Методы/ } \\
\text { Methods }\end{array}$ & $\begin{array}{l}\text { Хронометраж } \\
\text { трудоемкости } \\
\text { производственн } \\
\text { ой деятельности }\end{array}$ & $\begin{array}{l}\text { Метод экспертных } \\
\text { оценок (поэтапная } \\
\text { переоценка } \\
\text { результатов по по } \\
\text { формуле Байеса }\end{array}$ & $\begin{array}{l}\text { Квалиметрический } \\
\text { метод }\end{array}$ & $\begin{array}{l}\text { Экспертных } \\
\text { оценок, } \\
\text { анкетирование }\end{array}$ \\
\hline $\begin{array}{l}\text { Этапы/ } \\
\text { Stages }\end{array}$ & $\begin{array}{l}\text { 1. Этап. } \\
\text { Выявление или } \\
\text { уточнение } \\
\text { состава } \\
\text { компетенций }\end{array}$ & $\begin{array}{l}2 \text { Этап. Выбор или } \\
\text { разработка новой } \\
\text { технологии } \\
\text { (методики) } \\
\text { подготовки, } \\
\text { обеспечивающей } \\
\text { формирование } \\
\text { выявленных } \\
\text { компетенций }\end{array}$ & $\begin{array}{l}\text { 3. Этап. } \\
\text { Разработка } \\
\text { критериев } \\
\text { показателей } \\
\begin{array}{l}\text { оценки } \\
\text { компетенций }\end{array} \\
\end{array}$ & $\begin{array}{l}\text { 4. Этап. } \\
\text { Оценка } \\
\text { степени } \\
\text { предрасполож } \\
\text { енности к } \\
\text { виду } \\
\text { профессионал } \\
\text { ьной } \\
\text { деятельности }\end{array}$ \\
\hline Результат & $\begin{array}{l}\text { Компетентностн } \\
\text { ая } \\
\text { модель }\end{array}$ & $\begin{array}{l}\text { Технология } \\
\text { организации } \\
\text { целевой подготовки }\end{array}$ & $\begin{array}{l}\text { Оценочная } \\
\text { матрица }\end{array}$ & $\begin{array}{l}\text { Аттестат } \\
\text { готовности } \\
\text { (предрасполо } \\
\text { женности) }\end{array}$ \\
\hline
\end{tabular}

На каждом из выделенных основных этапов имеется возможность выполнения более мелких, соответствующих структуре решаемых задач.

Предполагается, что дополнительные профессиональные программы (ДПП) целенаправлены на формирование специализированных профессиональных компетенций (СПК), (см. Таблицу 3), обеспечивающих выполнение конкретных профессиональных задач, прописанных в целевом договоре о подготовке специалистов, когда формируется компетентностная модель специалиста, удовлетворяющая его современному портрету.

Учитывая значительность финансовых затрат на подготовку инженерных кадров (внебюджетное обучение стоит не менее 90000 руб/г), ошибиться с формированием профессиональных компетенций не желательно, требуется гарантия качества целевой подготовки инженерных кадров. Это позволяет обеспечить третья стратегема осуществление действий по совершенствованию научно-методического аппарата за счет подбора оптимального сочетания методов на каждом этапе проектирования ДПП и обоснованного принятия решений на каждом методологическом уровне проектирования. 
Используемая трехуровневая методология проектирования имеет свойственные каждому уровню характеристики:

- целевой уровень предусматривает подбор дидактических механизмов выявления и уточнения состава специализированных профессиональных компетенций (СПК));

- функционально-технологический уровень содержит дидактические механизмы и методы выбора технологии (методики) профессиональной подготовки, обеспечивающей готовность студентов к выполнению требований целевой подготовки;

- оценочный уровень предусматривает решение двух задач: разработку критериев и показателей для оценки СПК и оценки уровня профессиональной предрасположенности студента к виду профессиональной деятельности, его ориентацию на конкретный вид деятельности (конкретное производство).

Подбор оптимального сочетания методов, применяемых на каждом этапе проектирования ДПП целевой подготовки (см. Таблицу 4) позволяет сделать последовательность действий по проектированию обоснованной и обеспечивающей формирование требуемого портрета современного специалиста радиоэлектронного профиля.

В качестве четвертой стратегемы выступает использование при проектировании принципа динамической вариативности, заключающегося в том, что формулируется несколько альтернативных вариантов, которые подвергаются тщательной экспертизе. В результате определяется наилучший вариант, или отвергаются все, чтобы сформулировать новые. В качестве основного критерия приоритетности вариантов выступает оценка вероятности предложенных вариантов по формуле Байеса и переоценка выдвигаемых гипотез [4]. Таким образом, обеспечивается обоснованный выбор наиболее результативной технологии обучения (метода реализации программы) в условиях динамично меняющихся требований к уровню профессиональных компетенций выпускников [5].

Принцип динамической вариативности (соответствующая стратегема) использован нами для обоснования выбора концентрированного обучения, как основной формы подготовки студентов-целевиков по ДПП [4].

Каждый из альтернативных вариантов, как правило, обладает как достоинствами, так и недостатками. Перед вузом стоит задача выбора варианта, который позволит с наиболее высокой вероятностью решить задачу формирования требуемых образовательным стандартом и дополнительным договором компетенций. Для снижения вероятности ошибок при оперативном принятии решении обозначенных проблем в качестве отдельной пятой cmpaтегемы выделяем методику выбора приоритетного варианта (форм обучения, технологии/методики обучения) и построения алгоритма принятия решений, используя итерационный алгоритм последовательного привлечения экспертов и байесовский подход для переоценки результатов экспертизы [6].

Возможность с некоторой вероятностью наметить наиболее эффективные педагогические траектории состоит в применении методологии форсайта, заключающейся в обобщении разрозненно существующих экспертных позиций (в том числе путем «сталкивания» экспертов друг с другом) [3]. Учитывая то, что каждый эксперт в своем заключении не бывает абсолютно уверенным в принятии решении, можно говорить только о вероятности принятия им определенного решения, которое он может самостоятельно оценить, т. е. указать приоритетное место проекта и доверительную вероятность принятого им решения. Экспертная оценка может осуществляться и одновременно несколькими экспертами, а результаты затем проходить статистическую обработку. В нашей практике оказался более приемлемым вариант последовательного привлечения экспертов, когда их число можно оптимизировать. Алгоритм выбора приоритетного варианта среди выделенных подробно рассмотрен нами в работе [4], на конкретном примере. Он подтверждает нашу главную идею о том, что процесс проектирования ДПП необходимо рассматривать как 
последовательность стратегем, обеспечивающих оптимальное по качеству достижение поставленной цели.

Таким образом, представленный нами процесс разработки ДПП включает в себя последовательность следующих действий-стратегем: выделение этапов проектирования, использование на каждом этапе трехуровневой методологии (цель-технология-оценка), совершенствование методики на основе методов динамической вариативности и выбора наилучшего варианта с использованием вероятностных математических методов.

Исходной информацией для содержательного наполнения программ являются запросы экономики, производства и реальные условия их реализации на конкретном предприятии, жизненные интересы контингента обучаемых. В плане представленных идей разработаны, утверждены академическим сообществом (в рамках реализации целевой программы «Новые кадры ОПК») и реализуются в вузе около 20 образовательных модулей, среди которых «Разработка интеллектуальных средств и комплексов радиосвязи, «Проектирование интеллектуальных систем радиоэлектронной борьбы», «Проектирование и эксплуатация современных мехатронных систем на предприятиях ОПК» и др.

Выполнение условий целевых договоров, как и достижение запланированных результатов образования проверяется нами ежегодно на основе критериев комплексной оценки качества образования выпускников вуза.

\section{Обсуждения и заключения}

Подводя итоги вышесказанному, можно отметить, что процесс проектирования ДПП творческий процесс, встраивания профессионально-педагогических действий по формированию новых, экспериментально выявленных компетенций в действующую структуру государственных образовательных программ. Наиболее рационально такую работу следует осуществлять поэтапно, опираясь на выявленные стратегемы как инструменты, позволяющие обеспечить требуемый уровень качества проектирования ДПП целевой подготовки. Данное исследование завершает целый цикл работ авторов [3; 4; 6], являясь его логическим продолжением по тематике проектирования ДПП, позволяющим сделать выводы о том, что современные образовательные программы должны отвечать запросам динамично развивающегося общества, в котором все большее внимание уделяется акцентам целевой подготовке специалистов, воплощающей идеи интеграции науки, образования и производства. Практическая значимость исследования заключается в возможности использования данной методики разработки ДПП целевого обучения специалистов для наукоемких производств оборонно-промышленного комплекса, функционирующих в режиме их постоянного обновления. Предложенные рекомендации по организации проектирования образовательных программ в технических вузах России будут способствовать формированию образовательных компетенций у профессорскопреподавательского состава и производственных компетенций у обучающихся. Результаты проведенного исследования могут быть полезны как для специалистов в области образования, так и для работников промышленных предприятий. Дальнейшим развитием проведенных исследований может стать разработка системы автоматизации выбора содержания ДПП (из универсальных) на основе анализа требований предприятия-заказчика.

\section{Список литературы}

1. Александров Д. А., Тенишева К. А., Савельева С. С. Мобильность без рисков: образовательный путь «в университет через колледж» // Вопросы образования. 2015. № 3. C. 66-91. DOI: 10.17323/1814-9545-2015-3-66-91.

2. Блохин В.А., Козлов А.И., Муромцев Д.Ю., Орлова Л.П. Динамическая вариантность (альтернативность) при управлении проектами // Вестник ТГТУ. 2003. Т. 9. № 3. C. $390-405$. 
3. Волков А.Е., Кузьминов Я.И., Реморенко И.М., Рудник Б.Л., Фрумин И.Д., Якобсон Л.И. Российское образование - 2020: модель образования для инновационной экономики// Вопросы образования. 2008. №1 С. 32-64.

4. Дорохова Т.Ю., Пучков Н.П. Байесовский подход к проблемам определения приоритетности педагогических проектов// Continuum. Математика. Информатика. Образование. 2019. № 3 (15). С. 30-35.

5. Дорохова Т.Ю. Региональная система целевой подготовки специалистов для ОПК// Право и образование. 2019. № 6. С. 53-58.

6. Пучков Н.П., Дорохова Т.Ю. Проектирование системы концентрированной практикоориентированной подготовки специалистов для высокотехнологичных производств //Alma mater. 2018. № 2. C. 52-57.

\title{
STRATEGIES DESIGN TARGET TRAINING PROGRAMS AT A MODERN UNIVERSITY
}

\author{
T.Yu. Dorokhova $\mid$ Tambov State Technical University \\ Dr. Sci. (Pedagogy), associate professor \\ tandor81@mail.ru \\ Tambov \\ N.P. Puchkov \\ Dr. Sci. (Pedagogy), professor \\ puchkov_matematika@mail.ru \\ Tambov
}

\begin{abstract}
The article deals with the use of dynamic variability methods in the design of additional professional programs (APP) at a university that provides specialists' target training. The design process is presented as a sequence of actions for step-by-step indepth study of situations and positions of all subjects interested in the results. The purpose of the article is to offer specific recommendations on the theory and practice of APP design in the conditions of continuous change of production tasks based on the analysis of modern production problems.

Materials and methods. The results of the enterprises employees' survey revealed organizational and educational problems of improving the specialists' target training quality and outlined ways to resolve them. Expert assessment methods and probabilistic methods were used to make recommendations.

Results. The authors found that ensuring the target training quality in higher education is achieved by developing a APP based on: splitting the design process into certain stages; using a multi-level methodological design structure; the principle of dynamic design variability; an iterative algorithm for consistently attracting experts and a Bayesian approach to re-evaluate the results of expertise in order to develop a reasonable optimal solution.

Discussions and Conclusions. The development of educational programs should meet the needs of a dynamically developing economy, embodying the idea of integrating science, education and production. The practical significance of the research is to ensure the possibility of using the proposed methodology for developing APP specialists' target training for high-tech industries of the military-industrial complex, operating in the mode of their constant updating. The proposed recommendations will contribute to the formation of educational competencies in the teaching staff and industrial competencies in students and can be useful both for specialists in
\end{abstract}


educational field and for employees of industrial enterprises. The development of a system for automating the design process based on the use of new information technologies can become the development of the research.

Keywords: target training, additional professional programs strategies, methodology for the phased design of education.

\title{
References
}

1. Alexandrov, D. A., Tenisheva, K. A., Savelyeva, S. S. (2015). Mobility without risks: the educational path "to the university through college" [Mobil'nost' bez riskov: obrazovatel'nyj put' «v universitet cherez kolledzh»]. Educational Studies, 3, 66-91. (In Russ., abstract in Eng.) DOI: 10.17323 / 1814-9545-2015-3-66-91.

2. Blokhin, V.A., Kozlov, A.I., Muromtsev, D.Yu., Orlova L.P. (2003). Dynamic variability (alternative) in project management. [Dinamicheskaya variantnost' (al'ternativnost') pri upravlenii proektami]. Bulletin of the Tambov State Technical University, 9(3), 390 - 405. (In Russ., abstract in Eng.)

3. Dorokhova, T.Yu., Puchkov, N.P. (2019). Bayesian approach to the problems of determining the priority of pedagogical projects. [Bajesovskij podhod $k$ problemam opredeleniya prioritetnosti pedagogicheskih proektov]. Continuum. Maths. Informatics. Education, 3(15), 30-35. (In Russ., abstract in Eng.)

4. Puchkov, N.P., Dorokhova, T.Yu. (2018). Designing of a system of concentrated practiceoriented training of specialists for high-tech industries. [Proektirovanie sistemy koncentrirovannoj praktiko-orientirovannoj podgotovki specialistov dlya vysokotekhnologichnyh proizvodstv]. Continuum. Maths. Informatics. Education, 2, 52-57. (In Russ., abstract in Eng.)

5. Volkov, A.E., Kuzminov, Y.I., Remorenko, I.M., Rudnik, B.L., Frumin, I.D., Yakobson L.I. (2008) Russian education - 2020: a model of education for innovative economy [Rossijskoe obrazovanie - 2020: model' obrazovaniya dlya innovacionnoj ekonomiki]. Educational Studies, 1, 32-64. (In Russ., abstract in Eng.)

DOI: $10.24888 / 2500-1957-2020-3-35-42$

\section{УДК $\mid$ РУССКИЙ МАТЕМАТИЧЕСКИЙ ЯЗЫК ДЛЯ СТУДЕНТОВ- 378.14 ИНОСТРАНЦЕВ (НАЧАЛЬНЫЙ ЭТАП). ЧТЕНИЕ МАТЕМАТИЧЕСКИХ ТЕКСТОВ: ОДИН И ЕДИНИЦА}

Татьяна Ивановна Кузнецова

д.п.н., профессор

kuzti45@gmail.com

г. Москва
Московский государственный университет им. М.В. Ломоносова

\begin{abstract}
Аннотация. В статье предлагается исследование, проводимое на учебных материалах, разработанных в Институте русского языка и культуры МГУ имени М.В. Ломоносова для студентов-иностранцев. Статья является логическим продолжением начатого автором методического исследования: Русский математический язык (начальный этап). Чтение математических текстов: ноль и нуль [1]. Настоящей статье демонстрируется многообразие использования знака
\end{abstract}

